\title{
The VLT Interferometer Data Flow System: From Observation Preparation to Data Processing
}

\author{
P. Ballester ${ }^{1 \mathrm{a}}$, A.M. Chavan ${ }^{\mathrm{a}}$, A. Glindemann ${ }^{\mathrm{a}}$, C. Guirao ${ }^{\mathrm{a}}$, W. Jaffe ${ }^{\mathrm{b}}$, \\ P. Kervella ${ }^{\mathrm{a}}$, M. Peron ${ }^{\mathrm{a}}$, B. Pirenne ${ }^{\mathrm{a}}$, P.J. Quinn ${ }^{\mathrm{a}}$, A. Richichi ${ }^{\mathrm{a}}$, C.Sabet ${ }^{\mathrm{a}}$, \\ M. Schöller ${ }^{\mathrm{a}}$, A. Wicenec ${ }^{\mathrm{a}}$, R. Wilhelm ${ }^{\mathrm{a}}$, B. Wiseman ${ }^{\mathrm{a}}$, M. Wittkowski ${ }^{\mathrm{a}}$, S. Zampieri ${ }^{\mathrm{a}}$ \\ ${ }^{a}$ European Southern Observatory, Karl-Schwarzschildstr. 2, D-85748 Garching, Germany; \\ ${ }^{b}$ Leiden Observatory, Niels Bohr Weg 2, NL-2333 CA Leiden, The Netherlands
}

\begin{abstract}
In this article we present the Data Flow System (DFS) for the Very Large Telescope Interferometer (VLTI). The Data Flow System is the VLT end-to-end software system for handling astronomical observations from the initial observation proposal phase through the acquisition, processing and control of the astronomical data. The Data Flow System was first installed for VLTI first fringes utilising the siderostats together with the VINCI instrument and is constantly being upgraded in phase with the VLTI commissioning. When completed the VLT Interferometer will make it possible to coherently combine up to three beams coming from the four VLT $8.2 \mathrm{~m}$ telescopes as well as from a set of initially three $1.8 \mathrm{~m}$ Auxiliary Telescopes, using a Delay Line tunnel and four interferometry instruments. Observations of objects with some scientific interest are already being carried out in the framework of the VLTI commissioning using siderostats and the VLT Unit Telescopes, making it possible to test tools under realistic conditions. These tools comprise observation preparation, pipeline processing and further analysis systems. Work is in progress for the commissioning of other VLTI science instruments such as MIDI and AMBER. These are planned for the second half of 2002 and first half of 2003 respectively. The DFS will be especially useful for service observing. This is expected to be an important mode of observation for the VLTI, which is required to cope with numerous observation constraints and the need for observations spread over extended periods of time.
\end{abstract}

Keywords: Very Large Telescope, Interferometer, Data Flow System, Data Handling

\section{INTRODUCTION}

The Data Flow System ${ }^{1}$ is the VLT end-to-end software system for handling astronomical observations from the initial observation proposal phase through the acquisition, processing and control of the astronomical data. During the first period since the VLTI first fringes in March 2001 and with the 8.2 meter Unit Telescopes in October 2001, the system has been used intensively for the verification of the technical and science performance of the VLTI and the commissioning instrument VINCI. The operations model of the VLT allows principal investigators to apply for visitormode or service-mode observation programs. In visitor mode, the astronomer is present at the telescope and can adapt the observation program to specific target properties, changing observation conditions, or calibration needs. In servicemode the observatory scientific operators perform the observations and the data are processed and sent to the requesting astronomer. The service mode ${ }^{4}$ has been supported at the VLT since the beginning of operations with the first telescope ANTU in 1998. Most observations in interferometry will involve measurements for different spatial frequencies and are likely to require different configurations and spread over periods of several weeks. It is therefore expected that service observation will be a dominant mode at the VLTI.

With a maximum baseline length of 202 meters, the VLT Interferometer makes it possible to reach high angular resolution of the order of a few milliarcseconds. In March 2001, first fringes ${ }^{2}$ have been obtained with the VLT Interferometer using the test instrument $\mathrm{VINCI}^{3}$. Three more instruments will be active at the VLT Interferometer,

${ }^{1}$ pballest@eso.org; phone +49 8932006 435; fax +49 8932023 62; http://www.eso.org; European Southern Observatory, KarlSchwarzschildstr. 2, D-85748 Garching, Germany 
providing capabilities for coherent combination in the mid-infrared wavelength domain with MIDI, up to three nearinfrared optical beams with AMBER, and simultaneous interferometric observations of two objects with PRIMA. The coming VLTI science instruments like MIDI and AMBER make it necessary to handle large data volume of several tens of gigabytes per night and will set very high requirements in terms of pipeline computation speed. In this article we address the preparation of observations for an interferometry system, as well as data archival and processing.

\section{THE DATA FLOW CONCEPT}

The VLT Data Flow System has been in place since the opening of the first VLT Unit Telescope in 1998 and has been continuously evolving since then ${ }^{1}$. The procedure for proposal preparation in the Data Flow System ${ }^{4}$ involves a Phase I and a Phase II proposal preparation. In Phase I, proposals are submitted electronically to ESO and evaluated by the Observing Program Committee (OPC). After the OPC selection has taken place, Phase II preparation is based on the creation of Observation Blocks. Each Observation Block specifies an individual observation sequence which can be scheduled and executed completely without interruption. Observation Blocks are created by specifying the template parameters, target information, and user-defined scheduling constraints. The user will be assisted in these phases by a User Support Astronomer and by observation preparation tools. These tools include generic systems like finding chart generators or guide star selection systems, and instrument related tools like exposure time calculators (ETCs). Feasibility checks of the proposals are performed by the observatory and include technical feasibility and exposure time control.

The Observation Blocks (OBs) are queued for service-observing and organized in a schedule managed on a long-, medium-, and short-term basis. In Service Mode the constraint set defined by the user during phase 2 preparation is compared to the ambient data during the execution of observations, hence providing a better flexibility regarding the optimal execution of an observation. The other mode of observation is the Visitor mode, during which the astronomer attends at the observatory the execution of observation and makes use of the on-line pipeline.

Upon execution of the OBs, the data produced are stored first in an on-line archive system at Paranal observatory, then in the VLT archive in Garching. The Science Archive stores all raw frames produced by the instruments, as well as reference calibration data, and log files including maintenance and ambient conditions logs. The Science Archive is available to archive researchers and astronomers for catalog access and retrieval of scientific data as they become available after the end of the proprietary period, as well as retrieval of calibration data as soon as they have been processed and verified by the Data Flow Operations. A data reduction pipeline is available at the telescope for immediate quality assessment of the data, and in Service mode the data are reprocessed in Garching before being controlled by a Quality Control Scientist and being sent to the user.

\section{VERY LARGE TELESCOPE INTERFEROMETER}

The VLT Delay Lines form an essential part of the VLT Interferometer ${ }^{5}$ (VLTI). In order to enable a coherent combination of the light beams from the individual telescopes of the VLT (that is, to produce interferometric fringes at the focal point), the optical path differences (OPD) must be compensated by the Delay Line system to a precision in the order of a fraction of the coherence length. Each VLTI Delay Line consists of a retro-reflector mounted on a moving carriage. The optical design of this "Cat's Eye" is of the Ritchey-Chretien type that reflects the light very effectively. For this particular application, the "Cat's Eye" is not a corner cube with 3 perpendicular mirrors as is the case in the reflectors on cars and bicycles. It is in fact a telescope with a variable curvature mirror at the focus that sends a light beam back in a direction parallel to the one it came from. The moving base enables the Cat's Eye to travel along a 60 meters long rail track, thereby providing optical path difference corrections of up to 120 meters, as required for the VLTI array configurations at Paranal.

Although the ultimate sensitivity of the VLTI will be obtained when combining the VLT 8.2-m telescopes, the Auxiliary Telescopes (ATs) constitute an essential element of the VLTI for the following reasons:

- The ATs will make it possible to perform full testing and commissioning of the second generation of VLTI instruments, without having to make use of valuable light from the 8.2-m telescopes. 
- The ATs will provide the best imaging capability of VLTI by complementing the array of the four 8.2-m telescopes. The ATs can be placed on any of 30 possible stations and therefore provide many interferometric baselines. This will make interferometric imaging possible.

- The ATs provide the longest possible baseline of the VLTI (202 meters), fully utilizing the restricted space available on the Paranal mountain platform

- The ATs will enable full time use of the VLTI facilities. They are 100\% dedicated to VLTI, while the 8.2-m UTs will be only intermittently available for interferometric observations.

- The ATs do not need adaptive optics correction in the mid-infrared at 10 microns and are nearly corrected in the near-infrared.

To commission the Cerro Paranal VLTI complex even before the availability of ATs, ESO decided to build two dedicated light collectors (the VLTI siderostats) and a specific beam combiner instrument (VINCI). The latter is based on the proven concept of FLUOR (Fiber Linked Unit for Optical Recombination) which has been operated since 1995 as a focal instrument of the IOTA interferometer in Arizona. Later, it was decided to extend the capabilities of VINCI to provide an artificial star and an alignment verification unit. This extended instrument is called LEONARDO da VINCI $(\mathrm{LdV})$, while the name VINCI is still used in the beam combiner part.

MIDI will be the first scientific instrument installed at the VLTI. It will cover the mid-infrared range between 10 and 20 microns. It records spectrally dispersed fringes and can reach at $10 \mu \mathrm{m}$ a resolution of the order of 20 milliarcsecond. The expected limiting magnitude is $\mathrm{N}=5 \mathrm{mag}$ (400 mJy) in self-fringe-tracking mode, with $8 \mathrm{~m}$ telescopes and tip/tilt correction, and $\mathrm{N}=11.5 \mathrm{mag}(1 \mathrm{mJy})$ with external fringe tracking The actual design of MIDI is optimized for operation at $10 \mu \mathrm{m}$ and a possible extension to $20 \mu \mathrm{m}$ is considered. The main scientific objectives are very embedded young stars and protostars, the study of circumstellar disks, the search for exoplanets, the study of brown dwarfs, active galactic nuclei dust tori and the center of our own galaxy.

AMBER will combine up to three beams to be the first VLTI instrument with some imaging capability. It delivers spectrally dispersed fringes covering the three near-infrared bands J, H, K. Three spectral resolutions of approximately $35,1000,10000$ are supported. By analysing three beams at once it is possible to obtain images through phase closure techniques eliminating the influence of atmospheric turbulence on fringe position. The system can also be used in differential interferometry mode in order to estimate the phase difference between two spectral channels. The wavelength coverage will be extended in a second phase down to $0.6 \mu \mathrm{m}$ at the time the ATs become operational. The magnitude limit of AMBER is expected to reach $\mathrm{K} \cong 20$ when a bright reference star is available and $\mathrm{K} \cong 14$ otherwise. The main scientific objectives are the investigation at very high angular resolution of disks and jets around young stellar objects, active galactic nuclei dust tori, the search for exoplanets, the study of stellar properties such as diameter, pulsation, mass loss, with a spectral resolution up to 10000 .

The objective of PRIMA is to enable simultaneous interferometric observations of two objects - each with a maximum size of 2 arcsec - that are separated by up to 1 arcmin, without requiring a large continuous field of view. PRIMA can be subdivided into five sub-systems that are positioned in different locations of the VLTI. PRIMA is composed of a star separator that feeds two arbitrary objects into the Delay Lines, a laser metrology system to monitor the internal OPD between object and reference star, a differential Delay Line to adjust the OPD between object and reference star, a fringe sensor unit, and an astrometry detector allowing the observation of the fringe patterns of both stars on the same detector.

\section{OBSERVATION PREPARATION TOOLS}

Preparing an interferometric observation requires adequate tools that can handle the geometrical configuration of the array and target/calibrator positions. Most observations in interferometry will involve measurements for different spatial frequencies and are likely to require different configurations and spread over extended periods of time, several weeks or several months. The geometrical constraints on the observation of the science and calibrator targets and the limited observability of the objects due to both the range of delay lines and shadowing effects will make it necessary to assess the technical feasibility of observations at both stages of phase 1 and phase 2 preparation. During phase 1 , general tools 
like the WEB-based visibility calculator and exposure time calculators will be provided. In phase 2, the details of the observation can be validated more accurately.

\section{VLTI Visibility Calculator}

The VLTI Visibility Calculator is the tool used for such calculations. The VLTI Visibility Calculator computes the fringe visibility as a function of the object diameter, the position of the target in the sky at the time of the observation, and the selected configuration. It takes into account the horizon map of the observatory and shadowing effects induced by telescope domes and structures on the observatory platform. It computes the optical path length, the optical path difference, and takes into account the range of the Delay Lines to estimate the period of observation for a given target. With the longest VLTI baseline $(202 \mathrm{~m})$, angular resolutions can be measured at the scale of one milliarcsec $(1 \mathrm{mas})$. Unresolved objects, namely objects much smaller than the 1 mas limit, will yield maximal visibility. The fringe visibility decreases with the angular size of the observed target.
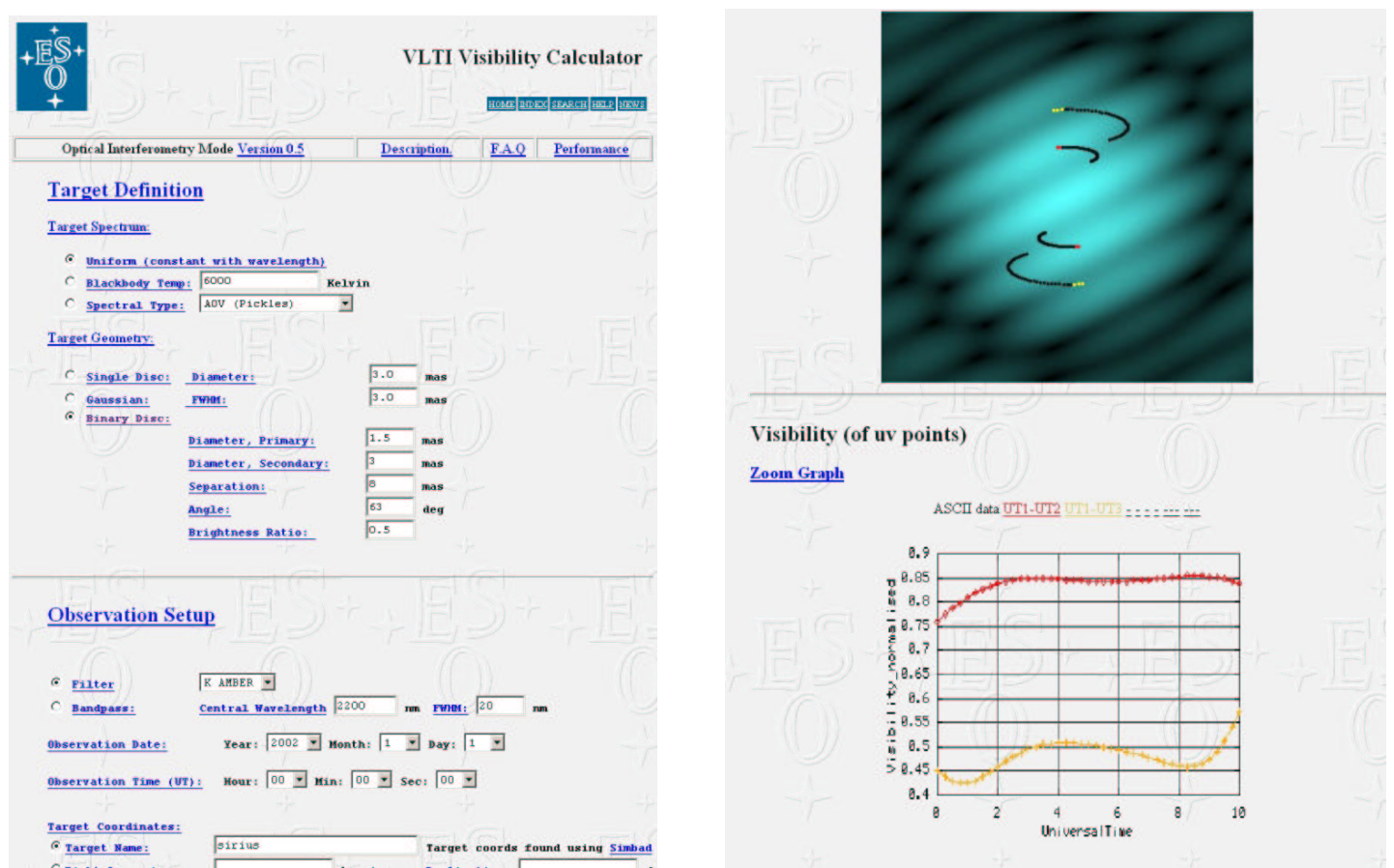

Figure 1: The VLTI Visibility Calculator. The VLTI Visibility Calculator is an Internet application similar to the ESO Exposure Time Calculators.

A prototype of the VLTI Visibility Calculator is shown in Figure 1. The current prototype has been initially defined in collaboration with the Jean-Marie Mariotti Center $^{6}$ (JMMC) supported by INSU (CNRS and Ministère de la Recherche, France). The prototype is used to identify an efficient and user-friendly procedure for dealing with the numerous interferometric observation constraints. The user-interface of the VLTI Visibility Calculator uses the same technology as the ESO Exposure Time Calculators (ETCs). It will therefore be an Internet tool like the existing VLT ETCs. An important feature of this Web-based prototype is that the user is provided not only with a visibility map of the source under consideration, but also with the so-called $\mathrm{u}-\mathrm{v}$ tracks for the chosen VLTI configuration. This enables the user to see immediately which parts of the visibility map can be studied and thereby estimate the usefulness and impact of such measurements. 


\section{VLTI Exposure Time Calculators}

Observation preparation tools have been provided for the VLT instruments in the form of Exposure Time Calculators accessible over the Internet (http://www.eso.org/observing/etc). The system provides a uniform access to the ETCs provided for the different VLT instruments. Several solutions have been designed to make it possible to efficiently develop and maintain these applications. For instance HTML templates and dictionaries are used to generate the pages on the fly, a macro language is used for prototyping and a database system is utilised to simplify the adjustment of instrument characteristics.

The ETC takes into account the spectral energy distribution of an object, the transmission curves and dispersions of an instrument, as well as the instrument configuration to compute the signal-to-noise ratio and inversely the exposure time required to obtain a given signal-to-noise ratio on the fringes. The ETC interface consists of two main pages, the input page and the result page. On the input page, the instrument setup and the target of the observations are defined. An HTML form is used to forward this information to a model application, which then performs the calculations and generates the result page. The result page summarises the input parameters and presents the results of the calculations, such as detected number of electrons of the object and sky, as well as various other information. The main result is the signal to noise for the observation, or alternatively, the number of integrations needed to achieve a specified signal-tonoise. In interferometry the signal to noise computations are significantly different from those required for a standard image or spectrum as done so far in the ETCs.

\section{PERFORMING INTERFEROMETRY OBSERVATIONS}

Preparing and executing an interferometric observation involves successive stages, each with specific constraints. In order to assess the technical feasibility of an interferometric observation one needs adequate tools to model the complete interferometer behaviour and to take into account for example shadowing effects, or the range of the delay lines. A typical suite of actions involved in the preparation of an interferometric observation involves the following steps:

- An assumption must be made concerning the intensity distribution of the source. Usually this will be in the form of a model with a uniform or limb-darkened disk, a Gaussian profile, a multiple system or any other shape that can be analytically described or has been obtained by techniques like radiation transfer calculations. The final goal is then to constrain the object parameters by model fitting or image reconstruction.

- The Fourier transform of the source intensity distribution that is the complex visibility function, is calculated. A set of spatial frequencies is identified which is suitable to obtain the required information on the object. A configuration of the interferometer array is selected among the available ones that will make it possible to sample the Fourier space in the adequate domain of spatial frequencies.

- One must make sure that the target is visible from the VLTI platform by all telescopes involved in the observation, taking into account possible shadowing effects. Shadowing effects can be caused for instance by the Unit Telescopes which obscure parts of the sky as seen by the Auxiliary Telescopes.

- The optical path difference must be estimated, in order to evaluate the total time that will be available for the observation.

- Suitable calibrators must be selected for the science object ${ }^{11}$. An interferometric calibrator is a target of (supposedly) known properties that will give a reference value for the measured visibility. Since the transfer function of the interferometer varies with time, calibrators and science objects must be observed alternatively within the finite stroke of the delay lines.

- The visibility amplitude (i.e. the modulus of the Fourier transform) of the target and calibrators can be estimated as well as the required exposure times. Note that in interferometry, it is not just the magnitude of the source that matters, but also the product of the total flux (in the resolution element) and the visibility. For example, between 
two sources of magnitude 1 and 2, and with visibilities 0.1 and 0.5 respectively, the former will look "fainter" to an interferometer than the latter.

- Once several series of targets and calibrators have been selected, they must be organised in a schedule of observations, where one will try to optimise the time spent in acquiring data taking into account priorities and observational constraints.

- In the Data Flow system, the instrument parameters are grouped in high level structures called Templates and Observation Blocks. The Template activates a standard operation mode of an instrument, for instance for the purpose of internal calibration, observation of calibration objects, or scientific observations. The Template signature includes the list of instrument parameters. Templates are grouped in Observation Blocks, which in the Data Flow correspond to an unbreakable unit of observation. For the interferometry, a prototype template has been tested which integrates the acquisition and observation of science and calibrator targets. This concept should make it possible to keep track of related observations in the archive and for pipeline processing.

- Templates and Observation Blocks are assembled with P2PP, the Phase 2 Proposal Preparation tool. This Java tool provides a uniform graphical user interface to all VLT and VLTI instruments. In visitor mode the OBs are loaded using P2PP directly at the VLTI. In service mode, the Observation Blocks are ingested in a repository in Garching.

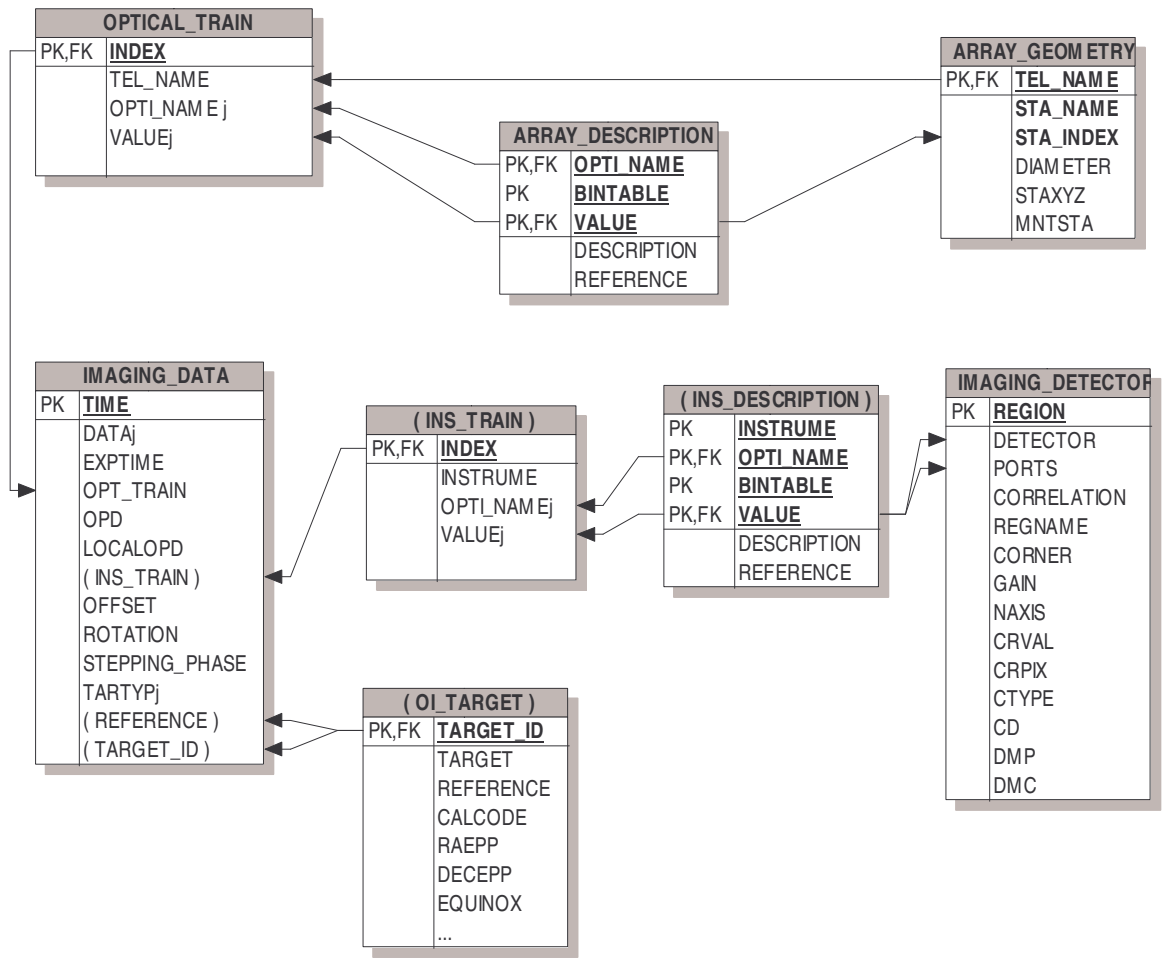

Figure 2: Data model for the VLT Interferometer. 


\section{INTERFEROMETRY DATA FORMAT}

Interferometry data are usually stored in the form of binary tables, since in most cases only a fraction of the detector is used to read the data. Several discussions are still taking place in the interferometry community for the definition of a general data structure within the FITS standard ${ }^{8}$. The header of the data follows the ESO Data Interface Control guidelines and provides the FITS keywords necessary for the data handling, processing, archive retrieval and proper documentation of the data.

The VLTI Interferometry tables are cross-referenced as illustrated in Figure 2. The ARRAY_DESCRIPTION table provides the description and bibliographical references to the indexes used in the ARRAY_GEOMETRY and OPTICAL_TRAIN tables. The INS_DESCRIPTION table provides the description and bibliographical references to the indexes used in the INS_TRAIN table. The ARRAY_GEOMETRY table describes the geographical information about the interferometer array. The OPTICAL_TRAIN table describes the configuration of the VLTI optical elements up to the instrument entrance window. The INS_TRAIN table describes the configuration of the instrument optical elements. The IMAGING_DETECTOR describes the detector settings and the IMAGING_DATA table contains the detector data.

\section{DATA STORAGE AND HANDLING}

The hardware system handling the VLTI data is similar to the system installed at the Unit Telescopes. Figure 3 shows the logical structure of the VLTI DFS system at Paranal: the instrument data (raw data) are transferred from the instrument workstation to the On-Line Archive System machine, where they are kept on a Redundant Array of Independent Disks (RAID) system. The Data Handling Server takes care of distributing the data to the pipeline, offline and archive workstations. Two machines are reserved for archive operations. One is dedicated to the Sybase server, where operations critical information is stored in various databases. The other Archive Storage (ASTO) machine is dedicated to media production, in particular CDs and DVDs.

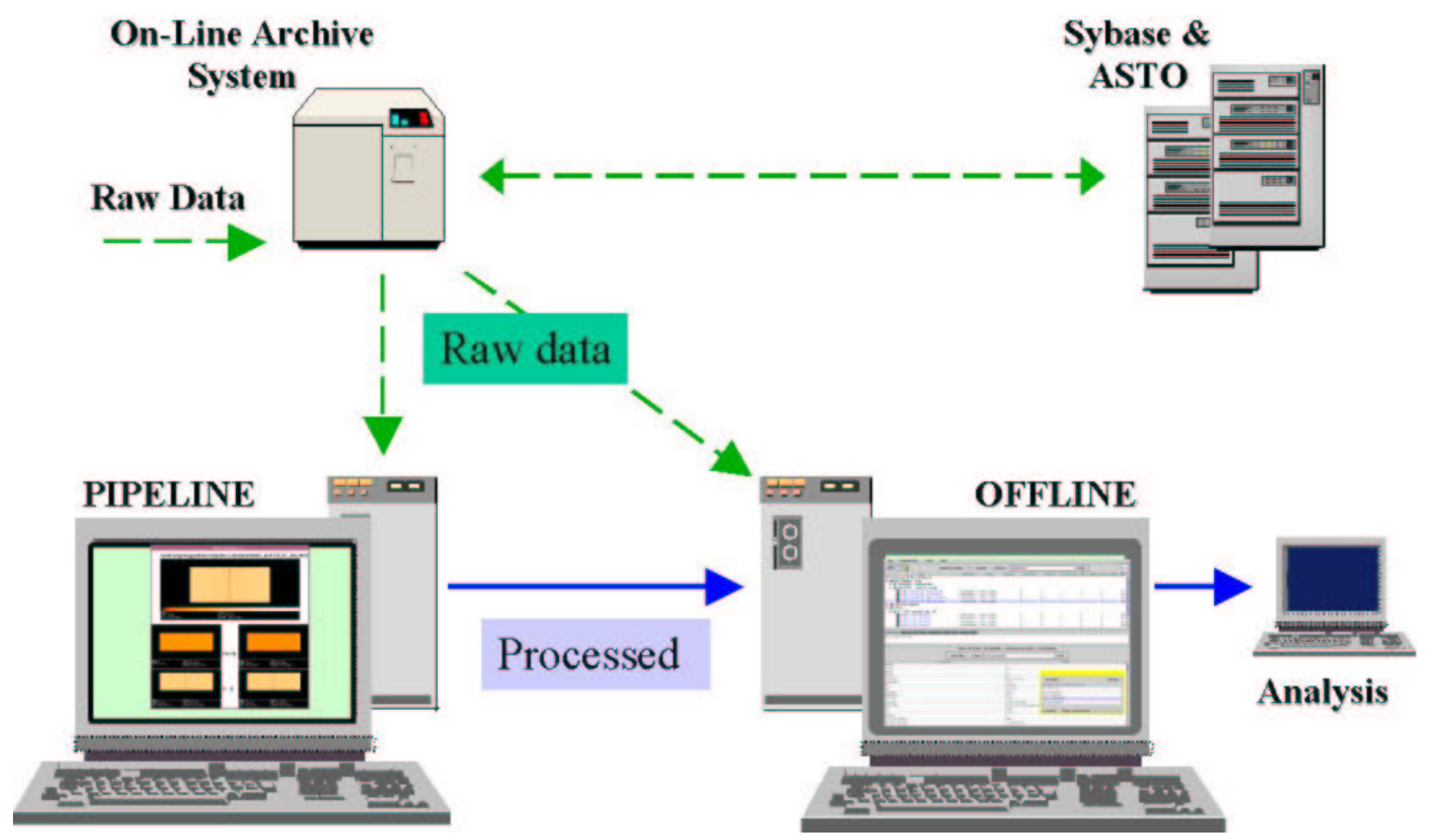

Figure 3: The VLTI Data Flow System at Paranal Observatory 
The pipeline and offline workstations have similar configurations and store the most recent raw and processed data as well as processing and displaying the data. The pipeline workstation usually runs in automatic mode, receiving and processing the data, displaying the results and transferring the processed data to the offline workstation. On the offline workstation, interactive tools are available for reprocessing and analysing the data for the purpose of commissioning and scientific evaluation.

The data rate for the first VLTI instrument VINCI is less than 1 gigabyte per night. The data rates will be higher for $\operatorname{AMBER}(0.7 \mathrm{MB} / \mathrm{s})$ and MIDI $(2.3 \mathrm{MB} / \mathrm{s})$. For MIDI this translates into more than 40 gigabytes of data per night.

The handling of such data volumes pushes the current system to its limits, in terms of overall throughput. For example, the DVD production is limited by the media capacity and writing speed. For this reason a new archive technology, based on magnetic disks rather than DVDs, is being evaluated and seems quite promising ${ }^{9}$.

\section{VINCI DATA REDUCTION PIPELINE}

The present VINCI pipeline performs automatically the first stage of processing and prepares FITS files containing the raw visibilities and all the information required for model fitting. The pipeline generates several data products. First the photometry-corrected interferograms are delivered for the purpose of commissioning analysis. This is delivered in ASCII form for further processing. Second, the raw visibilities, together with all the information required for further astrophysical analysis are written in a FITS file.

The pipeline receives the raw data frames from the On-Line Archive system and classifies them in accordance with the specifications given in the pipeline reduction rules. Conditions are evaluated on FITS keywords in order to classify the data. This determines the reduction procedure applied to the data. Before executing the procedure it may be necessary to read additional FITS keywords and to query auxiliary calibration data and tables from the pipeline local calibration database. A reduction block is prepared and sent for execution to a data reduction system. The VINCI measurement set consists of 4 values. These include the Interferometer 1, Interferometer 2, Photometer A and Photometer B measurements at a given time, also corresponding to a given Optical Path Difference (OPD). A scan is a sequence of OPD variation; it typically lasts about 0.1 second with VINCI. Series of scans are taken for different optical configurations: off-source scans, telescope A on-source only, telescope B on-source only, and a longer sequence of interferometric scans with both telescopes on-source. An exposure with VINCI includes the set of 4 batches and all auxiliary information. Each exposure produces a FITS file containing all the information necessary to derive uncalibrated visibilities.

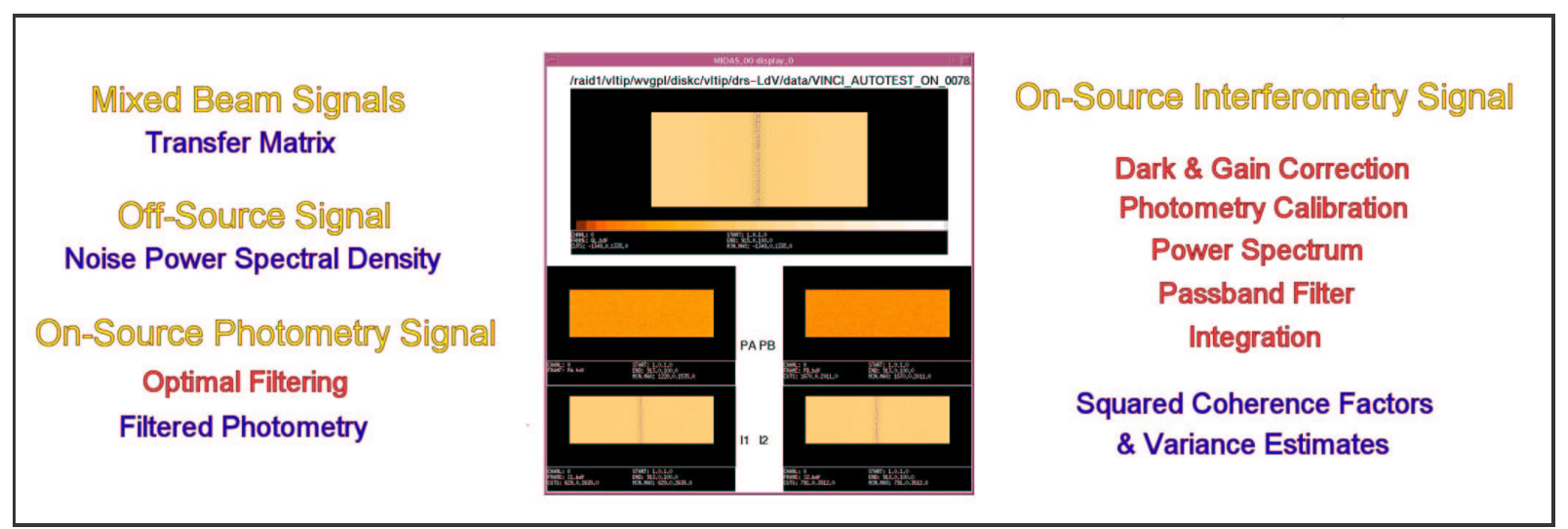

Figure 4: Main processing steps of the VINCI Data Reduction Software

The algorithm for the data processing ${ }^{7}$ is illustrated in Figure 4. The off-source signal is recorded with both light channels closed. This is processed to estimate the noise power spectral density. The mixed beam signals are recorded 
while the light passes through telescope A only or telescope B only. They are used to establish the throughput of the interferometer for each beam (transfer matrix) of the interferometer and to calibrate the intensity recorded on each interferometry output with respect to the two photometry signals. Once these preliminary calibrations are performed, it is possible to process the on-source signal. The on-source photometry signal is processed and filtered using information previously collected by the off-source and mixed beam signals. Finally the on-source interferometry signal is processed to yield squared coherence factors together with variance estimates. The processing involves corrections for the dark signal and transfer matrix, and integrating the power spectrum according to the spectral filter.

The calibration of visibilities of interferometry data involves two stages. First the raw visibilities are estimated on both the science and calibrator data. This is the most computing intensive step, during which science and calibrator data are processed in a similar way. Second, the calibrator information is used to estimate the instrument transfer function. This transfer function is then interpolated as a function of time. In interferometry a particular emphasis is placed on the statistical analysis of the data and the accurate estimation of the error bars. This second stage of calibration involves a limited amount of data as it applies to time averaged data. It may also require user interaction for the identification and rejection of calibration points. The calibrated visibilities with error estimates can then be used to determine the science object parameters.

\section{DATA QUALITY CONTROL}

The Quality Control (QC) system includes the tools used inside and outside the pipeline environment in order to control the conditions in which the data have been acquired and processed, in particular the instrumental and observational conditions. For VINCI the values tracked include in particular the signal to noise ratio on the photometric channels, the number of scans processed and rejected by the pipeline, the coefficients of the transfer matrix, the evolution of the transfer function and associated variance.

Quality control parameters are measured using the data and then written to the log files. The QC parameters have proven to be a very useful method for checking and tracking the health of instruments ${ }^{10}$. During the pipeline processing the data quantities characterising the performance of the instrument are measured. These values are written to operational $\log$ files. The $\log$ files are produced on a daily basis and can be used to produce automatic reports, graphs and summary information. The QC $\log$ files are used for diverse applications, for example to establish a catalog of observed objects, to produce trend graphs of the interferometer transfer function or to verify the quality of the processing by checking the number of scans rejected by the pipeline.

\section{OFF-LINE AND COMMISSIONING ANALYSIS}

For the scientific analysis of data, different interactive tools are provided on the off-line workstation, based on commercial data analysis packages. The data can be browsed and organised by Observation Blocks with the Gasgano tool, which provides means of organizing large amounts of data, classify them, view headers and call scripts on selected files. Gasgano can be used as a front-end graphical interface to the data reduction software.

Commands are provided to perform a second stage of calibration on the pipeline results. First the data are glued together and grouped by instrument modes. The calibration information can be tuned and the instrument transfer function is evaluated on the calibrator and interpolated with time. Finally it is possible to apply the calibration to science data and to model the intensity distribution of the source.

\section{ACKNOWLEDGEMENTS}

Many thanks to Bill Cotton from the National Radio Astronomy Observatory for important contributions to the data reduction package and the definition of the VLTI data format, to Gilles Duvert from Grenoble Observatory for helping with the VLTI visibility calculator using ASPRO. 


\section{ABBREVIATIONS}

\begin{tabular}{|l|l|}
\hline AMBER & Astronomical Multi-BEam Recombiner \\
ASTO & Archive Storage System \\
AT & Auxiliary Telescope (1.8m) \\
DFS & Data Flow System \\
DHS & Data Handling System \\
DVD & Digital Video Disc \\
ETC & Exposure Time Calculator \\
FITS & Flexible Image Transport System \\
FLUOR & Fiber Linked Unit for Optical Recombination \\
mas & milliarcsecond \\
MIDI & Mid-Infrared interferometric instrument \\
OB & Observation Block \\
OPC & Observing Program Committee \\
OPD & Optical Path Difference \\
OPL & Optical Path Length \\
P2PP & Phase 2 Proposal Preparation \\
PRIMA & Phase-Referenced Imaging and Microarcsecond Astrometry \\
QC & Quality Control \\
QC1 & Quality Control Level 1 \\
RAID & Redundant Array of Independent Disks \\
UT & Unit Telescope (8.2m) \\
VINCI & VLT INterferometer Commissionning Instrument \\
VLT & Very Large Telescope \\
VLTI & Very Large Telescope Interferometer \\
\hline
\end{tabular}

\section{REFERENCES}

1. Knudstrup. J., et al., Evolution and Adaptation of the VLT Data Flow System, , in Observatory Operations to Optimize Scientific Return III, SPIE Proc. 4844, 2002

2. Glindemann, A., et al., Light at the end of the tunnel - First fringes with the VLTI, ESO Messenger, 103, 2001

3. Kervella, P., et al., VINCI, the VLTI commissioning instrument: status after one year of operations, in Interferometry for Optical Astronomy II, , SPIE Proc. 4838-152, 2002

4. Grosbøl, P., Peron, M., The VLT Data Flow concept, in Astron. Data Anal. Soft. Syst. VI, ASP Conf. Series, 125, G. Hunt and H.E. Payne eds., 1997

5. Glindemann, A., et al., The VLT Interferometer: a unique instrument for high-resolution astronomy, in Interferometry in Optical Astronomy, SPIE Proc. 4006, 2--12, 2000

6. Duvert, G., Berio, P., ASPRO: A Software to Prepare Optical interferometry observations, SF2A-2001: Semaine de l'Astrophysique Francaise, in press.

7. Coudé du Foresto, V., Ridgway, S., Mariotti, J.M., Deriving object visibilities from interferograms obtained with a fiber stellar interferometer, Astron. Astrophys. Suppl. 121, 379-392, 1997

8. A Data Exchange Standard for Optical/IR Interferometry, NPOI/COAST, 15 March 2002, http://olbin.jpl.nasa.gov/iau

9. Wicenec, A., Knudstrup, J., ESO's archive computing framework, in Advanced Global Communications Technologies for Astronomy II, SPIE Proc. 4845-20, 2002

10. Ballester, P., et al., Data Quality Control and Instrument Modeling, in Observatory Operations to Optimize Scientific Return, SPIE Proc. 3349, 1998

11. Percheron, I., et al., Getting Ready for High Accuracy Measurements: The VLTI Calibrators Program, in Interferometry for Optical Astronomy II, SPIE Proc. 4838, 2002 